\title{
Privacidade para Crianças e Adolescentes em Redes Sociais Online sob a lente da Usabilidade: Um Estudo de Caso no Facebook
}

\section{Cristiana S. Silva ${ }^{1}$, Glívia A. R. Barbosa ${ }^{2}$, Ismael S. Silva ${ }^{2}$, Tatiana S. Silva ${ }^{3}$, Fernando H. Mourão ${ }^{4}$}

${ }^{1}$ Instituto de Ciências Exatas e Informática - Pontifícia Universidade Católica de Minas Gerais (PUC-MG). Av. Afonso Vaz de Melo, 1.200 - Barreiro de Baixo - Belo Horizonte MG - Brasil.

${ }^{2}$ Departamento de Computação - Centro Federal de Educação Tecnológica de Minas Gerais (DECOM/CEFET-MG). Av. Amazonas, 7675 - Nova Gameleira - Belo Horizonte, MG Brasil.

${ }^{3}$ Faculdade de Ciências Médicas de Minas Gerais. Alameda Ezequiel Dias, 275 - Belo Horizonte, MG - Brasil.

${ }^{4}$ Departamento de Ciência da Computação - Universidade Federal de São João Del Rey. Praça Frei Orlando, 170, Centro, São João del-Rei, MG - Brasil

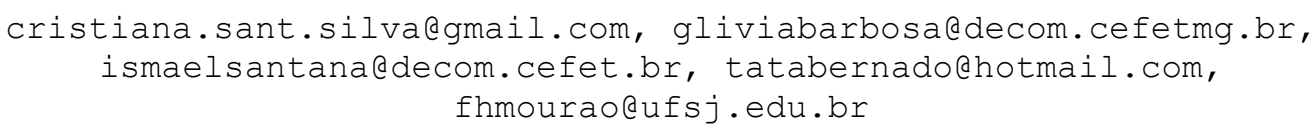

\begin{abstract}
This study aimed to characterize, through a case study on Facebook, such as children and adolescents deal with privacy features on online social networks and whether these resources are adequate for the use of this user's profile. The results indicated that there are usability violations that limit the use of the settings, by that user group that can compromise your privacy and security.

Resumo. Este trabalho buscou caracterizar, através de um estudo de caso no Facebook, como crianças e adolescentes lidam com os recursos de privacidade nas redes sociais online e se esses recursos estão adequados ao uso desse perfil de usuários. Os resultados indicaram que existem violações de usabilidade que limitam o uso das configurações, por parte desse grupo de usuário, que podem comprometer sua segurança e privacidade.
\end{abstract}

\section{Introdução}

Projetistas e pesquisadores da Computação e áreas relacionadas (e.g., [Junior et al., 2014],[Rodrigues et al., 2012],[Villela e Prates, 2015]) têm direcionado seus esforços para avaliar e/ou propor mecanismos de configuração de privacidade com o intuito de potencializar a segurança dos usuários dessas redes sociais online. Porém, alguns trabalhos têm demonstrado que os modelos de privacidade correntes nem sempre oferecem proteção 
adequada para os diferentes perfis de usuários, refletindo em uma disparidade entre a privacidade desejada e a real [Liu et al., 2011][Villela e Prates, 2015].

Conforme relatado por Villela e Prates (2015), essa disparidade ocorre, em tempo de interação do usuário com a rede social online, porque nem sempre a interface indica claramente aos vários perfis de usuários as formas e mecanismos para gerenciamento do nível de privacidade de seus dados. Em outras palavras, a usabilidade das configurações de privacidade pode não estar adequada ao uso dos diferentes tipos de usuários dessas redes [Chilana et al., 2012], sobretudo ao grupo formado por crianças e adolescentes [Fitton et al., 2014][Gilutz e Nielsen, 2002][Livingstone et al., 2011][Melo e Baranauskas, 2003].

Isso é um problema, porque apesar da existência de termos que indiquem o uso dessas redes sociais online para usuários acima de 13 anos [Boyd et al., 2011], estudos indicam que crianças e adolescentes têm utilizado esse tipo de sistema, na maioria das vezes, sem o acompanhamento dos responsáveis [Livingstone et al., 2011], reforçando a necessidade de também adequar os recursos para configuração de privacidade a esse tipo de usuário.

Dessa forma, torna-se relevante avaliar como a usabilidade dos controles de privacidade em redes sociais online reflete na percepção e uso que crianças e adolescentes fazem desses recursos. Isso porque, através dessa investigação será possível alertar sobre as vulnerabilidades que esse grupo de usuários pode estar sujeito, bem como melhorar a usabilidade dos mecanismos de privacidade existentes e/ou auxiliar no desenvolvimento de novas soluções que potencializem a privacidade de crianças e adolescentes nas redes sociais online [Fitton et al., 2014][Gilutz e Nielsen, 2002].

Nesse sentido, o objetivo desse trabalho consiste em avaliar e caracterizar como crianças e adolescentes têm lidado com as configurações de privacidade em seus perfis nas redes sociais online e se a usabilidade das funcionalidades que permitem tais configurações está adequada a esse perfil de usuários. Para isso foi realizado um estudo de caso no Facebook, considerando crianças e adolescentes do Brasil (i.e., usuários entre 6 a 17 anos, conforme definição do Art. $2^{\circ}$ da Lei $\mathrm{N}^{\circ} 8.069$ do Estatuto da Criança e do Adolescente $(1990)^{1}$ do Brasil).

O Facebook foi escolhido para o estudo de caso por ser a rede social online mais utilizada no mundo [Duggan et al., 2014][Duggan e Page, 2015] e porque, embora sua política de uso recomende sua utilização para maiores de 13 anos $^{2}$, dados como os apresentados por Boyd et al. (2011) apontam que crianças abaixo dessa idade fazem uso dessa rede social. Por sua vez, o estudo no Brasil se justifica, inicialmente porque o país é um dos campões no uso de redes sociais, em especial o Facebook que possui aproximadamente $30 \%$ de seus usuários no Brasil na faixa etária entre 6 e 17 anos [comScore, 2015][Consumer Reports News, 2013]. Outra motivação refere-se a estudos que evidenciam os perigos que esse grupo de usuários está sujeito no país (e.g., casos de

\footnotetext{
${ }^{1}$ Estatuto da Criança e do Adolescente do Brasil. Lei $\mathrm{N}^{\mathrm{o}}$ 8.069. 13 de Julho de 1990 . Available in: http://www.planalto.gov.br/CCIVIL_03/leis/L8069.htm.

${ }^{2}$ https://www.facebook.com/help/210644045634222
} 
pedofilia no Brasil através de redes sociais online [O tempo, 2014] e exposição de intimidades por parte de adolescentes com desconhecidos [Balmant, 2012]).

Os principais resultados indicaram como os problemas de usabilidade têm influenciado no grau de conhecimento e uso limitado que crianças e adolescentes fazem das configurações de segurança e privacidade no Facebook. Em suma, esse trabalho apresenta contribuições práticas e científicas para a área de sistemas colaborativos e afins, que não se limitam ao contexto do Brasil. Em termos práticos, alerta os projetistas de interface sobre a importância da usabilidade das configurações de privacidade de redes sociais online. De forma complementar, os resultados reportados podem servir de parâmetros para a melhoria e/ou criação de mecanismos de privacidade nesses ambientes colaborativos, com maior usabilidade, para crianças e adolescentes. Já em termos científicos, esse trabalho é relevante porque traz à tona a discussão sobre a importância de se propor e avaliar abordagens que visam o projeto e avaliação de interfaces para configurações de privacidade, em sistemas colaborativos, voltadas para crianças e adolescentes.

\section{Trabalhos Relacionados}

A privacidade em redes sociais online é uma questão complexa que tem sido explorada em diferentes aspectos. Dentre as possíveis linhas de investigação destacam-se os trabalhos que buscam caracterizar, de forma geral, a percepção e uso das configurações de privacidade nessas redes (e.g., [Albesher e Alhussain, 2013][Liu et al., 2011][Rodrigues et al., 2012]). Por exemplo, em Albesher e Alhussain (2013), os autores investigam a eficiência dos mecanismos de privacidade do Facebook para controlar a interação entre os usuários e discutem sobre a importância de se revisar regularmente esses controles, bem como as políticas de privacidade, de forma que permaneçam sempre adequados ao uso da diversidade de usuários que utilizam essa rede online.

De forma complementar, outras pesquisas buscam analisar a privacidade sob a lente de grupos de usuários segmentados como, por exemplo, crianças e adolescentes (e.g., [Boyd et al., 2011][Hinduja e Patchin, 2008][Souza e Dick, 2009]). Em seu trabalho, Boyd et al. (2011) alerta para as consequências de segurança e privacidade que as crianças estão sujeitas ao mentirem sobre a idade em redes sociais online, com a permissão dos pais. $\mathrm{O}$ estudo indica que muitos pais consentem com essa omissão, mas não acompanham devidamente o uso que suas crianças fazem da rede. Isso as tornam vulneráveis diante de pessoas maliciosas, comprometendo a segurança e privacidade das mesmas.

O trabalho realizado por Hinduja e Patchin (2008), analisou, de forma empírica, quais informações pessoais (e.g., nome completo, foto de perfil, escolaridade e telefone) que os adolescentes expõem no MySpace. Os resultados indicaram que, exceto em relação a foto de perfil, a maioria dos participantes são conscientes e menos de $20 \%$ expõem dados como nome completo, escolaridade e telefone. Contudo, alertam para o fato de que mais da metade desses adolescentes utilizam fotos pessoais para compor o perfil e essa informação é uma identificação visual que deve ser controlada.

De forma similar, Souza e Dick (2009) examinam quais informações as crianças compartilham no MySpace e qual o nível de compreensão delas sobre as questões de privacidade. Os autores concluíram que a quantidade de informações pessoais exposta por 
esse grupo de usuários está relacionada à, pelo menos, dois fatores: (1) o nível de conhecimento que elas têm sobre privacidade e (2) a adequação dos controles de segurança ao uso desse tipo de usuário. Isso porque os resultados sugeriram que as crianças que entendem melhor o conceito de privacidade e conseguem manipular a interface do MySpace, sem dificuldades, estão menos propensas a divulgar informações pessoais nessa rede online. Sendo assim, Hinduja e Patchin (2008) e Souza e Dick (2009) reforçam que análises como estas são necessárias não apenas para crianças e adolescentes, mas para outros perfis de usuários, de forma a alertar para as vulnerabilidades que eles podem estar sujeitos ao expor informações pessoais.

Uma vez que a percepção sobre a privacidade em redes sociais pode ser influenciada também pela cultura, diversos estudos têm procurado entender como pessoas de diferentes países lidam com essa questão (e.g., [Dey et al., 2012][Krasnova e Veltri, 2010][Tsoi e Chen, 2011]). Um exemplo é a pesquisa realizada por Tsoi e Chen (2011), na qual, através do uso de questionários e grupos focais, os autores apresentaram diferenças significativas entre os usuários de Hong Kong e da França em relação aos padrões de uso das redes sociais online e às suas respectivas configurações de privacidade.

Embora, diferentes aspectos relacionados à privacidade tenham sido investigados, não foram encontrados trabalhos que fizessem a análise proposta neste estudo, abordando a privacidade para crianças e adolescentes em redes sociais sob a lente da usabilidade. Além disso, o fato do presente artigo apresentar um estudo de caso no Facebook com crianças e adolescentes do Brasil, enraíza discussões significativas não apenas para o país, que é um dos campeões no uso das redes sociais online, mas também para toda a comunidade científica e para os profissionais que investigam e projetam aspectos relacionados a usabilidade e privacidade em redes sociais online. Isso porque o trabalho traz a tona como as questões de usabilidade podem impactar na percepção e uso que os usuários fazem dos controles de privacidade nessas redes.

\section{Metodologia}

Para atingir o objetivo proposto foi realizado um estudo de caso em três etapas. Inicialmente, foi realizada uma inspeção de usabilidade com base nas heurísticas propostas por Nielsen (1993). Assim, os recursos de privacidade do Facebook foram avaliados sob a perspectiva de especialistas em Interação Humano Computador (IHC). Essa análise ocorreu com o suporte de um profissional formado em Terapia Ocupacional especialista em psicopedagogia infanto-juvenil, a fim de constatar se essas funcionalidades são adequadas ao entendimento e uso de crianças e adolescentes do Brasil.

Posteriormente, foi realizada uma avaliação com esse grupo de usuários, através do método de entrevista semiestruturada, para detectar como eles utilizam esses recursos e o quão vulnerável eles estão nessa rede social. Finalmente, os resultados foram triangulados para que fosse possível verificar como a usabilidade das configurações de privacidade do Facebook tem impactado na segurança de crianças e adolescentes.

A triangulação refere-se a um procedimento da pesquisa qualitativa realizada através da comparação de dados extraídos por diferentes métodos, cujo intuito é conferir a validade dos resultados obtidos em uma pesquisa científica [Cho e Trent, 2006]. Neste 
caso, a validação consiste em buscar diferentes interpretações para a mesma questão de pesquisa [Cho e Trent, 2006]. A seguir os principais resultados do estudo de caso são apresentados.

\section{Usabilidade das Configurações de Privacidade do Facebook sob a Perspectiva de Especialistas}

Uma das formas de analisar a usabilidade de um sistema é executar a Avaliação Heurística (AH) que é um método qualitativo consolidado para avaliar interfaces [Nielsen, 1994]. Nessa etapa do trabalho, os passos da AH foram executados, com o objetivo de se identificar possíveis problemas de usabilidade nos mecanismos de privacidade do Facebook para crianças e adolescentes do Brasil.

A avaliação foi realizada na primeira quinzena de maço de 2015 por dois avaliadores com experiência na aplicação da $\mathrm{AH}$. Os resultados foram validados por um especialista na área de IHC (i.e., profissional da área com mais de sete anos de experiência) e por um Terapeuta Ocupacional, especializado em psicopedagogia e comportamento infanto-juvenil, com mais de cinco anos de experiência na área. A atuação do especialista em psicopedagogia contribuiu para que os especialistas em IHC pudessem justificar porque os problemas de usabilidade identificados impactavam na interação de crianças e adolescentes.

\subsection{Análise dos Potenciais Problemas Identificados}

Durante a avaliação, foi encontrado um total de 12 problemas nas configurações de privacidade que violaram pelo menos uma heurística de usabilidade. Desses, $83 \%$ foram classificados com a gravidade 3 (i.e., problema grande), $8 \%$ com gravidade 4 (i.e., catastrófico) e $9 \%$ com gravidade 2 (i.e., problema pequeno).

Uma vez que cada problema poderia ser associado à ausência de uma ou mais heurísticas de usabilidade e que uma mesma heurística poderia ser violada em diferentes problemas, para realizar a análise proposta e apresentar os resultados de forma consolidada, foram verificadas quantas heurísticas de usabilidade foram violadas e com que frequência (i.e., incidência) essas violações aconteciam. A Figura 1 apresenta os resultados consolidados.

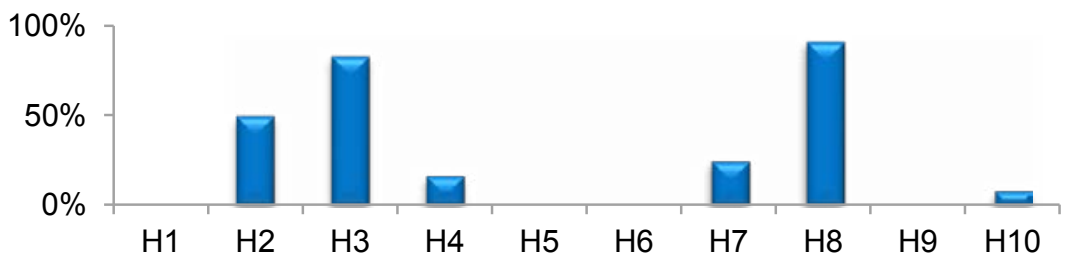

Figura 1. Violação das Heurísticas de Usabilidade nas Configurações de Privacidade do Facebook

Através desses dados, é possível notar que as heurísticas "H3 - Controle e liberdade do usuário", "H8 - Flexibilidade e eficiência de uso" e "H2 - Correspondência entre o sistema e o mundo real" tiveram grande percentual de violação, ou seja, foram violadas em diferentes problemas. A H8 teve o maior percentual de violação com 92\%, H3 teve o 
segundo maior percentual com $83 \%$ e $\mathrm{H} 2$ com terceiro percentual teve $50 \%$ do total de incidências nos problemas.

A frequência similar de problemas violando essas três heurísticas pode estar associada à relação existente entre as mesmas. Isso porque, se o projetista não faz uso de elementos em sua interface que façam analogia ao mundo real, dificilmente o usuário consegue interagir com a interface de uma forma eficiente, e ao se deparar com uma interface pouco flexível o usuário se sente desmotivado no uso das funcionalidades. Tais problemas podem comprometer a percepção e uso dos mecanismos de privacidade do Facebook, uma vez que o usuário pode se sentir limitado e, consequentemente, desmotivado em aplicar tais configurações em seu perfil [Nielsen, 1994][Nielsen, 1993][Gilutz e Nielsen, 2002].

Levando em consideração o grupo foco desta pesquisa, ao lidar com crianças e adolescentes esses violações se tornam ainda mais grave, pois esse grupo é mais impaciente para lidar com essas dificuldades durante as configurações de privacidade [Fitton et al., 2014][Gilutz e Nielsen, 2002]. Tudo isso diminui as possibilidades desses usuários se protegerem dos perigos que a rede pode oferecer em relação aos outros usuários.

As demais heurísticas, H1 - Visibilidade e status do sistema; H4 - Consistência e padrões; H5 - Ajuda aos usuários a reconhecer, diagnosticar e recuperar-se de erros; H6 Prevenção de erros; e H7 - Reconhecimento ao invés de memorização, apresentaram um percentual de violação inferior a $10 \%$.

\section{Apreciação da Privacidade no Facebook sob a Perspectiva de Crianças e Adolescentes}

Com o objetivo de investigar a percepção de crianças e adolescentes sobre a privacidade no Facebook e verificar se os problemas de usabilidade levantados na etapa anterior estavam sendo vivenciados por esses usuários, foi realizada uma avaliação por meio de entrevista semiestruturada composta por 30 questões. As questões foram agrupadas de forma que fosse possível identificar: (1) o perfil do participante da pesquisa; (2) detalhes da conta e amigos do Facebook; (3) o conteúdo que é publicado; e (4) conhecer como lidam com a privacidade na rede.

A entrevista ocorreu na segunda quinzena do mês de março de 2015, com crianças e adolescentes brasileiras do estado de Minas Gerais. A mesma foi conduzida por três autores desse trabalho, dois especialistas em IHC e um Terapeuta Ocupacional especializado em psicopedagogia infanto-juvenil. Assim como na avaliação por inspeção (i.e., primeira fase da metodologia desse trabalho), a atuação do especialista em psicopedagogia foi de extrema importância para auxiliar tanto os participantes, quanto os especialistas em IHC na condução e análise dos dados da entrevista, de forma a manter o foco da avaliação na percepção e uso das configurações de privacidade do Facebook por crianças e adolescentes. Ao todo 42 pessoas participaram dessa etapa da pesquisa.

\subsection{Perfil dos Participantes}

Dos 42 participantes da avaliação, a maioria (75\%) foi classificada na faixa etária entre 15 e 16 anos, $9 \%$ declararam ter 10 anos, enquanto que $8 \%$ têm 11 anos e $8 \%$ têm 14 anos. A 
divisão de gêneros dos participantes foi $75 \%$ do sexo feminino e $25 \%$ do sexo masculino. Em relação à formação, contatou-se que a maioria dos participantes $(58 \%)$ está cursando o ensino médio, $25 \%$ concluiu apenas o ensino fundamental e $17 \%$ está cursando o nível fundamental. Esses dados indicam que o grupo participante dessa avaliação possui um grau de formação suficiente para compreender e discernir sobre a questão de privacidade no Facebook levantada nesse trabalho.

Os participantes foram questionados sobre as redes sociais online que utilizam atualmente e/ou já utilizaram. A Figura 2 sumariza esses dados e indica o Facebook como a rede social mais utilizada pelos participantes (100\%). Em relação ao tempo de utilização do Facebook, constatou-se que todos os participantes possuem conta nessa rede social há pelo menos 2 anos. Quanto à frequência de uso, os dados obtidos mostraram que todos os participantes acessam o Facebook pelo menos duas vezes por semana. Desses, 58\% acessam a rede social diariamente, através do uso de dispositivos móveis, por pelo menos 1 hora.

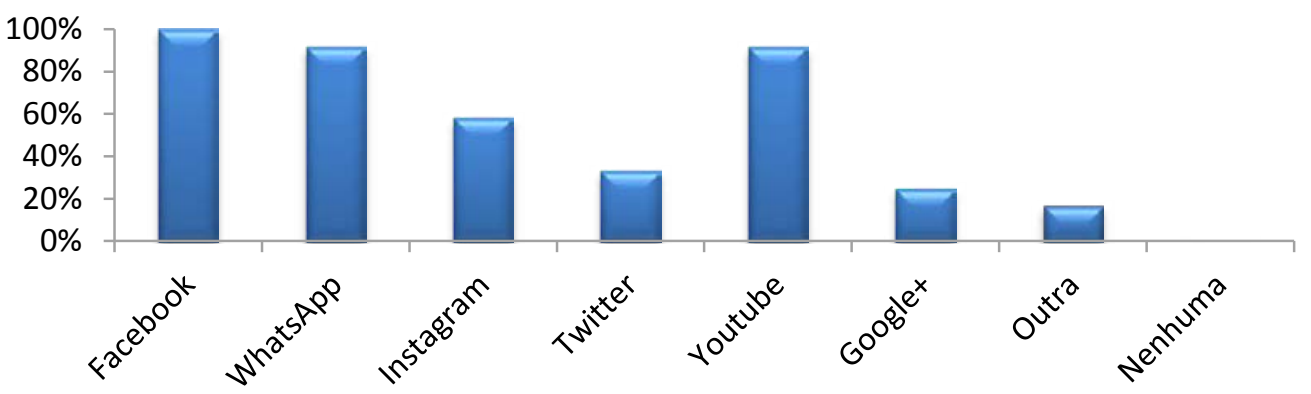

Figura 2. Redes Sociais Online utilizadas

\subsection{Contatos e Uso do Facebook}

Inicialmente, os participantes foram convidados a responder quantos amigos eles mantêm no Facebook. Os dados obtidos podem ser visualizados na Figura 3, que indicam que a maioria dos participantes dessa pesquisa (67\%) possui mais de 500 amigos no Facebook e $33 \%$ têm entre 201 a 500 contatos nessa rede social. Ao analisar essa quantidade de amigos distribuída pela idade dos participantes, como demonstrado na Figura 4, é possível perceber, por exemplo, que todos os entrevistados entre 10 e 11 anos possuem de 401 a 500 amigos no Facebook e todos os adolescentes participantes com idade de 14 anos estão conectados a mais de 500 pessoas.

Diante do elevado número de conexões estabelecidas no Facebook, os participantes foram questionados se conheciam pessoalmente todos os amigos que mantêm nessa rede social. 92\% afirmaram não conhecer pessoalmente todos os amigos do Facebook. Essa informação chama atenção para o fato de que pessoas mal intencionadas podem se aproveitar dessa situação para cometer crimes, como adquirir dados pessoais para cometer assédios e/ou abusos, ou até mesmo para humilhar ou fazer ameaças (e.g., cyberbullying). Essa questão se torna mais evidente quando, desses participantes, 25\% afirmam interagir ativamente (e.g., trocam mensagens de texto e fotos), através do Facebook, com esses contatos que eles não conhecem pessoalmente. 


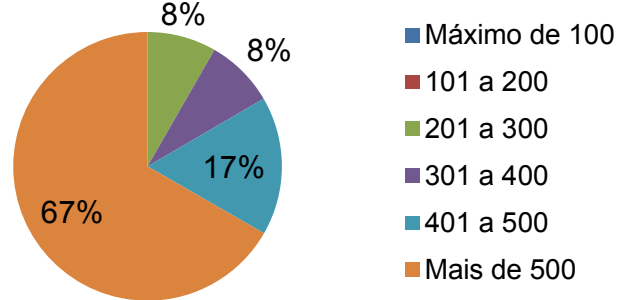

Figura 3. Total de Amigos no Facebook

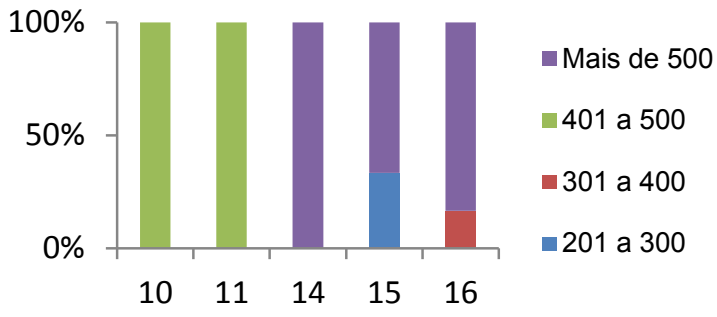

Figura 4. Idade versus Amigos no Facebook

Os dados apresentados até o momento servem de alerta para a questão de segurança e privacidade dessas crianças e adolescentes na rede. Isso porque um grande número de pessoas pode ter acesso aos dados e informações pessoais desses usuários, que representam um grupo mais frágil e suscetível a ser atraído e engando por pessoas maliciosas [Boyd et al., 2011][Dey et al., 2012][ Dey et al., 2013][Livingstone et al., 2011].

Tal observação reforça a importância de se garantir maior usabilidade para que crianças e adolescentes possam perceber e se sentir motivados a utilizar os mecanismos que permitem configurar privacidade no Facebook, uma vez que esse incentivo pode contribuir para minimizar o risco de exposição desse público.

Os participantes também foram questionados sobre o tipo de conteúdo que publicam no Facebook. Através da Figura 5 é possível constatar que $25 \%$ dos entrevistados admitem publicar dados pessoais, como nome, endereço e telefone. Fato que chama atenção para a falta de percepção de algumas crianças e adolescentes para o perigo que podem estar correndo ao expor esses dados, uma vez que os mesmos podem ser utilizados para prejudicá-los.

Além dos dados pessoais, os participantes também admitiram publicar conteúdo que expressam seu estado pessoal (e.g., alegrias, tristezas, conquistas, viagens) e fotos, o que os expõem ainda mais na rede online. Ao expor seu estado pessoal para qualquer pessoa, o usuário fica mais propenso a ações de criminosos que podem se aproveitar de um momento de fragilidade para se aproximar. Além disso, o fato de expor fotos pessoais facilita a ação desses criminosos ao visualizar suas vítimas, os lugares que frequentam ou até os bens pessoais que possuem [Boyd et al., 2011][Dey et al., 2012][ Dey et al., 2013][Livingstone et al., 2011].

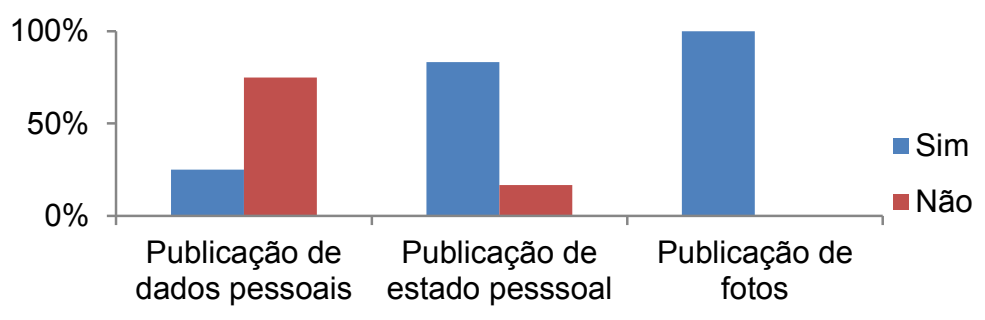

Figura 5. Conteúdos Publicados no Facebook

Esse comportamento se torna mais grave ao analisar a visibilidade dos conteúdos publicados. Verificou-se que embora metade dos participantes limite o acesso às publicações somente a amigos, $42 \%$ informaram que a visibilidade de conteúdos publicados 
em sua linha de tempo é pública, mesmo o Facebook oferecendo a possibilidade de configurar a visibilidade de cada conteúdo publicado. Além disso, $8 \%$ dos participantes disseram não saber qual é visibilidade do conteúdo publicado por eles. Mesmo sendo uma parcela pequena, essa informação é relevante, pois demonstra que ainda existem pessoas, dentro do perfil analisado nesta pesquisa, que não tem consciência sobre a existência do controle de visibilidade de conteúdos publicados. O uso limitado, ou até mesmo o desconhecimento, desse mecanismo de controle pode ser justificado pelo problema de usabilidade exemplificado na avaliação por inspeção (seção 4.1.).

Uma vez analisada a interação com os contatos do Facebook, bem como o tipo e visibilidade do conteúdo publicado pelos participantes, a avaliação com os usuários seguiu de forma a caracterizar: (1) a percepção e uso das configurações de privacidade por crianças e adolescentes e (2) problemas de privacidade já vivenciados por esses usuários. Os resultados são apresentados na próxima subseção.

\subsection{Privacidade no Facebook}

Inicialmente, os entrevistados foram questionados se estão preocupados com a segurança e privacidade no Facebook e se conhecem a política de privacidade dessa rede social. Dos participantes, $68 \%$ afirmaram se preocupar com sua privacidade, porém, $80 \%$ disseram não ter conhecimento (i.e., não leram) sobre a política que rege a privacidade no Facebook.

Essa informação pode ajudar a explicar o fato de que menos de $50 \%$ dos entrevistados afirmam conhecer e/ou utilizar todas as configurações de privacidade disponíveis no Facebook, conforme demonstrado na Figura 6 que lista os principais controles de privacidade oferecidos pelo Facebook e indica o percentual de participantes dessa pesquisa que conhecem e/ou utilizam cada um desses recursos.

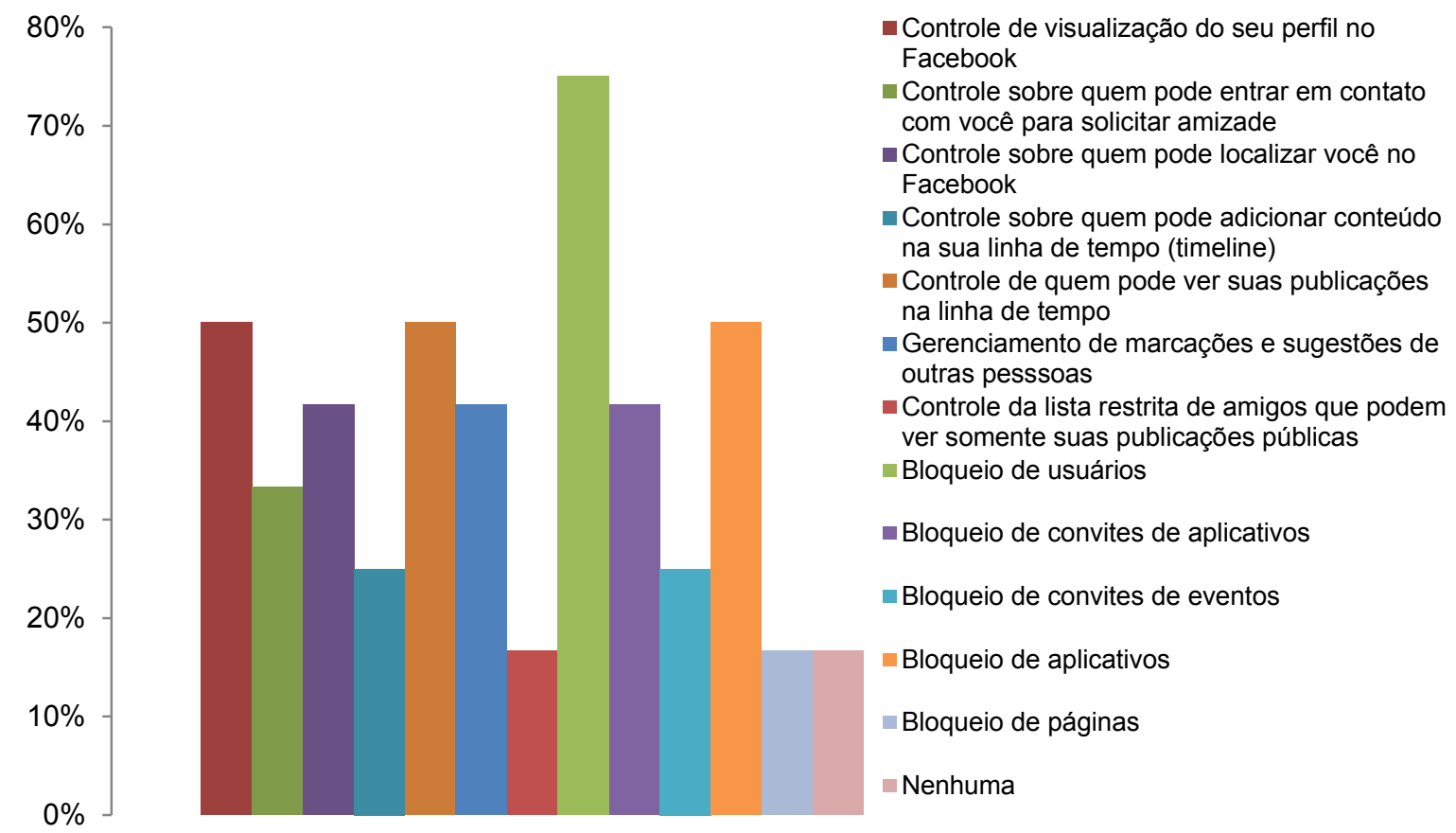

Figura 6. Configurações de Privacidade do Facebook utilizadas 
Através desses dados é possível verificar, por exemplo, que a configuração "Controle da lista restrita de amigos que podem ver somente suas publicações $e$ informações públicas", útil para limitar os conteúdos a um determinado grupo de amigos que não são de total confiança, é conhecida e utilizada por apenas $18 \%$ dos entrevistados. Outra configuração que é muito importante é a de "Controle sobre quem pode adicionar conteúdo na sua linha de tempo (timeline)". Essa também obteve baixo índice de conhecimento, apenas $25 \%$. Essa configuração permite restringir as pessoas que podem adicionar conteúdo na linha de tempo, o que previne o usuário de possíveis transtornos com conteúdos indesejáveis como insultos e ameaças.

Como informado anteriormente, os participantes foram questionados se já vivenciaram problemas de privacidade no Facebook (nesta questão, mais de uma violação poderia ser indicada). Dos entrevistados, como demonstrado na Figura 7, 50\% disserem ter sofrido algum tipo de violação de privacidade. Sendo que, $33 \%$ tiveram sua conta invadida e pelos menos $8 \%$ admitiram ter sofrido algum tipo de assédio e/ou abuso e problemas com contas falsas no Facebook. Ao contrastar a visibilidade do perfil dos participantes com o tipo de violação de privacidade, é possível concluir, com o auxílio da Figura 8, que as crianças e adolescentes que mantêm um perfil público sofreram diferentes tipos de violações de privacidade.

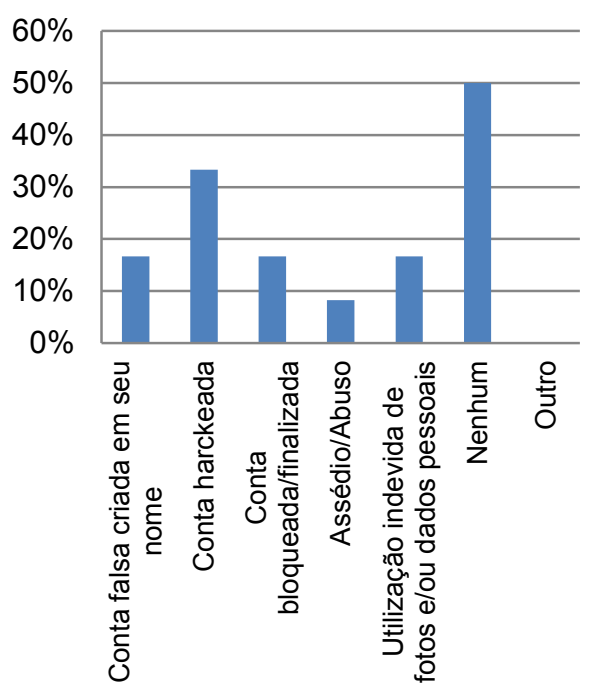

Figura 7. Violações de Privacidade sofridas

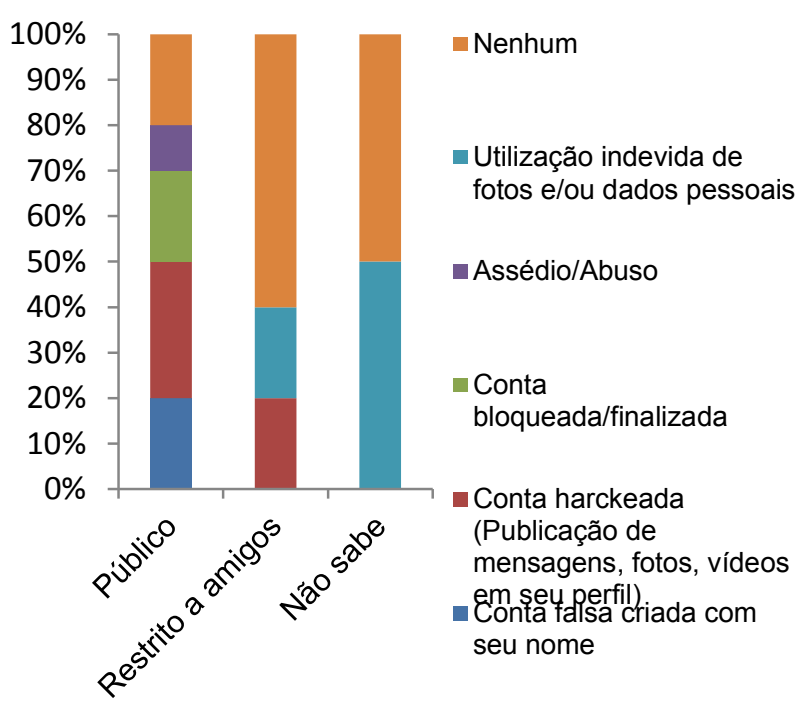

Figura 8. Visibilidade versus Violação

Finalizada as análises dessa etapa da metodologia foi possível observar que embora a maioria das crianças e adolescentes, participantes dessa avaliação, afirme preocupar com sua segurança e privacidade no Facebook, menos da metade conhece e/ou utiliza os principais controles disponíveis nessa rede social para se resguardar. Essa falta de conhecimento pode influenciar no uso limitado, ou até mesmo na não utilização, de importantes configurações de privacidade nessa rede social por esse público.

Esse uso limitado é um alerta, porque conforme informado anteriormente, crianças e adolescentes são potencialmente mais vulneráveis nessas redes e o fato de deixarem o perfil público, adicionarem e interagirem com desconhecidos e compartilharem informações 
pessoais como dados, fotos e "estados pessoais" aumentam, de forma significativa, as chances de violações de privacidades, como de fato ocorreu com $50 \%$ dos participantes.

Os resultados dessa avaliação chamam atenção não somente de pais e responsáveis, em relação aos cuidados que devem ser tomados durante o acesso de seus filhos, mas também dos desenvolvedores de redes sociais online, uma vez que a oferta de configurações de privacidade mais claras e adequadas ao uso desse público podem contribuir para aumentar a segurança e privacidade de crianças e adolescentes nessas redes.

\section{Triangulação e Discussão dos Resultados}

Para verificar como a usabilidade das configurações de privacidade do Facebook tem impactado na segurança de crianças e adolescentes do Brasil, a última etapa dessa pesquisa consistiu em triangular e discutir os resultados obtidos na avaliação de usabilidade por inspeção com os dados coletados durante a entrevista com o público alvo dessa pesquisa.

Os resultados apresentados indicam que, uma possível explicação para que a maioria dos participantes desse estudo de caso desconheça a política de privacidade do Facebook e esteja fazendo um uso limitado dos controles oferecidos por essa rede, é a violação das heurísticas "H2 - Correspondência entre o sistema e o mundo real", "H4 Consistência e padronização", "H7 - Reconhecimento em vez de memorização" e "H10 Ajuda e Documentação".

Ao violar H2, H4, H7 e H10, o Facebook não faz uso de elementos de interface compatíveis com a expectativa e experiência dessas crianças e adolescentes, fazendo com que eles encontrem dificuldades em reconhecer e utilizar as opções de configuração de privacidade, bem como em procurar auxílio através dos recursos de ajuda do sistema [Nielsen, 1993][Nielsen, 1994][Gilutz e Nielsen, 2002]. Tais dificuldades podem inviabilizar, ou até mesmo impedir, que as configurações de privacidade sejam aplicadas adequadamente.

Isso porque, uma vez que todas essas heurísticas buscam garantir a facilidade de uso, eficiência, produtividade e satisfação dos usuários, o fato de violá-las contribui para que crianças e adolescentes façam um uso limitado dos mecanismos de privacidade, já que eles não se sentem guiados e motivados em aplicá-las, devido às barreiras (i.e., dificuldades) encontradas na interface disponível para essas configurações.

Logo, essa triangulação evidencia que os problemas de usabilidade refletem no uso que crianças e adolescentes fazem dos recursos de privacidade do Facebook e a pesquisa mostrou que de fato o uso limitado compromete a segurança desses usuários. Isso porque $50 \%$ dos entrevistados admitiram ter problemas com violação de privacidade. Sendo assim, a má usabilidade dos recursos para configurar a privacidade aumenta a vulnerabilidade de crianças e adolescentes no Facebook diante de pessoas mal intencionadas.

\section{Conclusões e Trabalhos Futuros}

Este trabalho buscou apreciar e caracterizar como crianças e adolescentes têm lidado com os controles de privacidade em seus perfis nas redes sociais online e se a usabilidade desses controles está adequada a esse perfil de usuários. Para isso foi realizado um estudo de caso 
no Facebook, considerando crianças e adolescentes do Brasil, uma vez que esse país é um dos campeões no uso desse sistema que é a rede social mais utilizada no mundo. A análise aqui apresentada é importante porque, embora o uso das redes sociais online seja recomendado para maiores de 13 anos, pesquisas indicam que usuários abaixo dessa faixa etária têm utilizado esse tipo de sistema, na maioria das vezes, sem o acompanhamento dos responsáveis, reforçando a necessidade de também adequar os recursos para configuração de privacidade a esse público.

Os principais resultados apresentados e discutidos indicaram como os problemas de usabilidade têm influenciado no grau de conhecimento e uso limitado que crianças e adolescentes do Brasil fazem das configurações de segurança e privacidade no Facebook. Nesse sentido, embora o estudo de caso tenha sido realizado no Facebook, considerando o perfil de crianças e adolescentes do Brasil, os resultados apresentados e discutidos nesse trabalho são relevantes e não se limitam apenas ao contexto desse país. A relevância deste tipo de investigação pode ser sustentada pelo argumento apresentado por Wilson et al. (2009) e Willinger et al. (2010), no qual os autores listam os desafios de pesquisas relacionados a redes sociais online.

Segundo Wilson et al. (2009) e Willinger et al. (2010) para que seja possível oferecer soluções que enderecem as necessidades dos diferentes perfis de usuários que utilizam as redes sociais online, em todas as situações como, por exemplo, a privacidade, é preciso focar em aspectos dinâmicos e comportamentais durante o uso desse tipo de sistema e, sobretudo, apreciar e caracterizar como os diversos tipos de usuários estão interagindo atualmente, de forma a verificar e endereçar os problemas que podem estar afetando a qualidade dessa interação [Wilson et al., 2009] e [Willinger et al., 2010].

Sendo assim, este trabalho apresenta contribuições práticas e científicas/metodológicas para a área de sistemas colaborativos e afins. Em termos científicos, esse trabalho é relevante porque discute sobre a importância de se propor e avaliar abordagens que visam o projeto e avaliação de interfaces para configurações de privacidade voltadas para crianças e adolescentes, reforçando a relevância de iniciativas como: (1) User Experience (UX) para adolescentes [Fitton et al., 2014]; (2) Diretrizes de usabilidade para crianças [Gilutz and Nielsen, 2002]; e (3) Design de interface e interação para crianças [Melo and Baranauskas, 2003] no âmbito de sistemas colaborativos. Contudo, vale destacar que a relevância do trabalho não se limita a essa contribuição para as pesquisas na área, isso porque, a metodologia adotada neste estudo de caso pode ser reproduzida na avaliação de outras redes sociais, em relação à usabilidade dos recursos de privacidade, considerando outros perfis de usuários.

Já em termos práticos, o trabalho alerta os projetistas de interface sobre a importância da usabilidade nos controles de privacidade de redes sociais online. De forma complementar, os resultados reportados podem servir de parâmetros para a melhoria e/ou criação de mecanismos de privacidade nesse ambiente virtual, com maior usabilidade, para crianças e adolescentes.

Nesse sentido, como trabalhos futuros propõe-se a criação de um design com melhorias e/ou um conjunto de melhores práticas que descrevam os recursos de privacidade 
mais adequados para crianças e adolescentes. Além disso, outra direção futura consiste na avaliação dos mecanismos de privacidade em redes sociais online sob as perspectivas de outros perfis, como, por exemplo, pessoas com necessidades especiais e idosas.

\section{Referências}

Albesher, A. and Alhussain, T. 2013. Privacy and security issues in social networks: an evaluation of Facebook. In Proceedings of the 2013 International Conference on Information Systems and Design of Communication (ISDOC '13). ACM, New York, NY, USA, 7-10.

Balmant, O. 2012. Cresce exposição de jovens na internet. O Estado de São Paulo, Caderno Vida, p.27, 02 dez 2012. Estadão Geral

Boyd, D., Hargittai, E., Schultz, J. and Palfrey, J. 2011. Why Parents Help Their Children Lie to Facebook: Unintended Consequences of the 'Children's Online Privacy Protection Act'. First Monday, 16(11), November 7, 2011.

Chilana, P., Holsberry, C., Oliveira, F. and Ko, A. 2012. Designing for a billion users: a case study of facebook. In CHI '12 Extended Abstracts on Human Factors in Computing Systems (CHI EA '12). ACM, New York, NY, USA, 419-432.

Cho, J. and Trent, A. (2006). Validity in qualitative research revisited. Qualitative Research, 6(3):319--340.

comScore. 2015. Brazil Digital Future in Focus. Available in: $<$ https://goo.gl/H51ep1 >. Last access: Dec - 18, 2015.

Consumer Reports News. 2013. Facebook may let children under age 13 use the site. Available in: <http://goo.gl/tgqbI7> Last access: Jun - 07, 2015.

da Silva, S. R.; e Pereira, R. (2008). Aspectos da interação humano-computador na web social. In Proceedings of the IX IHC 2008, Outubro de 2008, pp.350-553.

Dey, R., Jelveh, Z. and Ross, R. 2012. Facebook users have become much more private: A large-scale study. In Proceedings of the 4th IEEE International Workshop on Security and Social Networking. IEEE, pp. 346-352.

Dey, R., Tang, C., Ross, K. W. and Saxena, N. 2012. Estimating age privacy leakage in online social networks. In Proceedings of the IEEE INFOCOM 2012, Orlando, FL, USA, pages 2836--2840, 2012.

Dey, R., Ding, Y. and Ross, K. W. 2013. Profiling high-school students with facebook: how online privacy laws can actually increase minors' risk. In Proceedings of the 2013 conference on Internet measurement conference (IMC '13). ACM, New York, NY, USA, 405-416.

Duggan, M., Ellison, N. B., Lampe, C., Lenhart, A. and Mary Madden, M. 2015. Social Media Update 2014. In Pew Internet and American Life Project. Available in: $<$ http://www.pewinternet.org/2015/01/09/social-media-update-2014/>, Last access: Dec $-18,2015$. 
Duggan, M., and Page, D. 2015. Mobile Messaging and Social Media 2015. In Pew Research Center. Available in: < http://www.pewinternet.org/2015/01/09/social-mediaupdate-2014/>, Last access: Dec - 18, 2015.

Fitton, D., Bell, B., Read, J. C., Iversen, O., Little, L. and Horton, M. 2014. Understanding teen UX: building a bridge to the future. In CHI '14 Extended Abstracts on Human Factors in Computing Systems (CHI EA '14). ACM, New York, NY, USA, 79-82.

Gill, A. J., Vasalou, A., Papoutsi, C., and Joinson, A. (2011) "Privacy Dictionary: A Linguistic Taxonomy of Privacy for Content Analysis". In Proceedings of CHI 2011, Session: Privacy May 7-12, 2011, Vancouver, BC, Canada.

Gilutz, S. and Nielsen, J. 2002. Usability of Websites for Children: Children (Ages 3-12) on the Web - 70 Design Guidelines. 3rd Edition. Nielsen Norman Group. Available in: <www.nngroup.com/reports/kids/>. Last access: Dec - 29, 2015.

Hinduja, S., and Patchin, J. W. 2008. Personal information of adolescents on the Internet: A quantitative content analysis of MySpace. Journal of Adolescence, 31(1), 125-146

Junior, M. P., Xavier, S. and Prates, R. O. 2014.Investigating the use of a Simulator to Support Users in Anticipating Impact of Privacy Settings in Facebook. In Proceedings of the 18th ACM International Conference on Supporting Group Work, ACM, 63-72.

Krasnova, H., and Veltri, N.F. 2010. "Privacy Calculus on Social Networking Sites: Explorative Evidence from Germany and USA", Proceedings of the 43rd Hawaii International Conference on System Sciences (HICSS), IEEE, 2010, pp. 1-10

Liu, Y., Gummadi, K. P. and Mislove, A. 2011. Analyzing Facebook Privacy Settings: User Expectations vs Reality. In Proceedings of Internet measurement conference, ACM, 61-70.

Livingstone, S., Ólafsson, K. and Staksrud, E. 2011. Social networking, age and privacy. EU Kids Online, London, UK. Available in: <http://eprints.lse.ac.uk/35849/>. Last access: Dec - 29, 2015.

Melo, A. M. and Baranauskas, M. C. 2003. Design with children: a Semiotic approach. In Proceedings of the Latin American conference on Human-computer interaction (CLIHC '03). ACM, New York, NY, USA, 69-78.

Nielsen., J. Usability engineering. San Francisco: Morgan Kauffman, 1993.

Nielsen., J. 1994. Usability inspection methods. In Conference Companion on Human Factors in Computing Systems (CHI '94), Catherine Plaisant (Ed.). ACM, New York, NY, USA, 413-414.

Nielsen., J. 2000. Why you only need to test with 5 users. 2000. Available in: < https://goo.gl/qik9ZN>. Last access: Dec - 29, 2015.

O TEMPO (Jornal). 2014. Pedófilo se passa por atriz de Chiquititas para abordar crianças. . Available in: < http://goo.gl/nXGJ4g > . Last access: Dec - 29, 2015. 
Pereira Junior, M., Xavier, S. I. de R. and Prates, R. O. 2014. Investigating the Use of a Simulator to Support Users in Anticipating Impact of Privacy Settings in Facebook. In Proceedings of the 18th International Conference on Supporting Group Work (GROUP '14). ACM, New York, NY, USA, 63-72.

Pesce, J. P., Casas, D. L., Rauber, G., and Almeida, V. 2012. Privacy attacks in social media using photo tagging networks: a case study with Facebook. In Proceedings of the 1st Workshop on Privacy and Security in Online Social Media (PSOSM '12). ACM, New York, NY, USA, Article 4, 8 pages.

Rodrigues, K. R. H., Canal, M. C., Xavier, R. A. C., Alencar, T. S., and Neris, V. P. A. 2012. Avaliando aspectos de privacidade no Facebook pelas lentes de usabilidade, acessibilidade e fatores emocionais. In Companion Proceedings of the 11th Brazilian Symposium on Human Factors in Computing Systems (IHC '12).

Souza, Z. D., and Dick, G. N. 2009. Disclosure of information by children in social networking-Not just a case of "you show me yours and I'll show you mine". Int. J. Inf. Manag. 29, 4 (August 2009), 255-261.

Tsoi, H. K., and Chen, L. 2011. "From Privacy Concern to Uses of Social Network Sites: A Cultural Comparison via User Survey. 2011. In Proceedings of the IEEE Third International Conference on Social Computing (SocialCom), pp.457-464, 9-11 Oct. 2011.

Villela, M. L. B., and Prates, R. O. 2015. Supporting Designers in Modeling Privacy for Social Network Sites. In Proceedings of 15th Brazilian Symposium on Human Factors in Computing Systems. (IHC'15). November 03-06, 2015.

Wilson, C., Boe, B., Sala, A., Puttaswamy, K. P. N., Zhao, B. Y. 2009. User interactions in social networks and their implications. In Proceedings of the 4th ACM European conference on Computer systems (EuroSys '09). ACM, New York, NY, USA, PP. 205218 (2009).

Willinger, W., Rejaie, R., Torkjazi M., Valafar, M., Maggioni, M. 2010. Research on online social networks: time to face the real challenges. SIGMETRICS Perform. Eval. Rev. 37, 3, pp. 49--54 (2010). 